\title{
Sensibilidade e aleitamento materno em díades com recém-nascidos de risco
}

\author{
Cristiane Alfaya \\ Universidade Luterana do Brasil, Santa Maria \\ Ligia Schermann \\ Universidade Luterana do Brasil, Canoas
}

\begin{abstract}
Resumo
O presente estudo investigou a relação entre o tipo de alimentação do bebê (aleitamento materno, fórmula e alimentação mista) e os comportamentos de interação mãe-bebê, a partir do conceito de sensibilidade materna. Participaram 20 díades com bebês nascidos pré-termos (Grupo I) que necessitaram de tratamento intensivo neonatal, 14 díades com bebês nascidos a termo (Grupo II) que necessitaram de tratamento intensivo neonatal, e 24 díades com bebês nascidos a termo sadios (Grupo III). O risco do recém-nascido foi considerado a partir de sua necessidade de tratamento intensivo neonatal. A interação mãe-bebê foi filmada durante a situação de face-a-face, aos dois meses de idade do bebê, e avaliada conforme o Protocolo de Interação Mãe-Bebê (Schermann et al. 1994) que pontua itens referentes aos comportamentos interativos da mãe, do bebê e da díade mãe-bebê. Os resultados mostraram que a sensibilidade materna diferiu entre os três grupos investigados ( $\mathrm{p}=0,039$ ), havendo maior incidência no Grupo III (grupo controle). A partir do estudo realizado, é possível inferir que a sensibilidade materna é mais favorável em mães de bebês nascidos a termo e sadios do que em mães de bebês nascidos com risco neonatal. $\mathrm{O}$ aleitamento materno é um importante fator que promove o estabelecimento de uma sensibilidade materna mais favorável, sendo fundamental o incentivo da amamentação mesmo para recém-nascidos pré-termo e a termo de risco.
\end{abstract}

Palavras-chave: sensibilidade materna; alimentação; nascimento de risco

\begin{abstract}
Breastfeeding and maternal sensitivity in dyads with infants born at risk. The purpose of this study was to investigate the relation between the kinds of infant's feeding (breastfeeding, complement and mixed) and the mother-infant interactional behaviors. The participants included 20 dyads of pre-term infant (Group I) who had needed intensive care, 14 dyads of full-term infant (Group II) who had needed intensive care, and 24 dyads of full-term health infants (Group III). The risk was considered by the neonatal intensive care needed. The mother-infant interaction was filmed at face-to-face situation and evaluated through Mother-Infant Interaction Protocol (Schermann et al. 1994) to assess the maternal behaviors, the infant behaviors and the dyads behaviors. Results showed that maternal sensitivity was very different between groups ( $\mathrm{p}=0.039$ ); Group III (control group) had a better maternal sensitivity. Therefore maternal sensitivity is better in mothers of full-term health infants, than in mothers of pre-term infant and full-term infant who had needed intensive care. Breastfeeding is an important factor to promote a better maternal sensitivity.
\end{abstract}

Keywords: maternal sensitivity; feeding; infant born at risk

$\mathrm{H}$ istoricamente, os temas da observação e análise das interações iniciais entre pais-bebê são recentes, com menos de cinqüenta anos de estudo. Na área da psicanálise, por exemplo, a observação direta das interações começou a ser desenvolvida na década de 1940, a partir dos estudos de René Spitz e Anna Freud com crianças separadas de seus pais, em instituições e sob condições de guerra. Os estudos realizados nessas condições de separação, em que a mãe não estava presente para atender a criança, puderam constatar a natureza da dependência infantil e a importância do cuidado materno para o desenvolvimento da criança. As descrições da severa patologia resultante da falta de cuidado materno incentivaram novos estudos acerca desse tema dos quais se pode citar Margaret Mahler e John Bowlby, que admitiram a importância das primeiras relações de cuidado, especialmente a qualidade do vínculo com o cuidador. 
John Bowlby, em 1958, introduziu o termo interação, ampliando a idéia de intercâmbio do bebê com a mãe. Considerava não apenas o aspecto da gratificação oral e redução da tensão enquanto satisfação de necessidades fisiológicas do bebê através da mãe, como se pensava até então, mas também passou a supor a presença do apego na criança como uma modalidade básica e inata do comportamento da espécie humana, assim como os comportamentos inatos de choro, sucção e sorriso. A ênfase de Bowlby na competência inata do recém-nascido para entrar em comunicação social com a pessoa que cuida dele e a concepção de Winnicott sobre a mãe e o filho como uma unidade influenciaram bastante os estudos de interação até os dias de hoje, e, além disso, passou-se a dar enfoque à atividade e aos comportamentos estimuladores, e não apenas ao desamparo e à passividade, sendo o bebê visto como um dos agentes da formação da relação entre pais e filhos, em que o bebê pode criar a sua própria gratificação, pois ele possui um papel ativo na relação, sendo inclusive dotado de certo grau de independência (Brazelton \& Cramer, 1992).

O conceito de sensibilidade materna foi desenvolvido por Ainsworth (1982), sendo definido como a habilidade da mãe em perceber, interpretar e responder adequadamente às necessidades e à comunicação do bebê, promovendo uma base segura para o desenvolvimento sócio-emocional da criança. Para tanto, é fundamental que esta mãe mostre-se atenta aos sinais de seu bebê, e principalmente sensível a estes sinais, sendo capaz de se colocar no lugar do bebê. É a partir do nascimento e através dos primeiros comportamentos interativos da mãe em relação ao bebê, e do bebê em relação à mãe que o vínculo afetivo entre mãe e filho é efetivamente estabelecido. A qualidade da relação mãe-bebê pode ser observada através dos comportamentos interativos da díade nas diversas situações, desde o simples brincar até o amamentar. Aos poucos, na medida em que esses comportamentos vão se repetindo, a criança começa a apresentar um determinado padrão de interação social, construindo uma representação a respeito de si mesmo e do ambiente, que poderá ser seguro ou ameaçador, de acordo com a qualidade das trocas interativas. A regularidade dos comportamentos também é considerada um importante fator para a construção de uma representação segura em relação ao ambiente.

É importante lembrar que a construção dos comportamentos interativos entre a mãe e o bebê recémchegado está diretamente relacionada aos padrões de interação construídos pela mãe desde a sua infância com a sua própria mãe ou cuidadora. A experiência de cuidado recebido pela mãe, certamente, influencia o sentimento de competência materno da mãe em relação ao seu bebê (Klaus, Kennell, \& Klaus, 2000). Dessa forma, o padrão de interação estabelecido pela díade mãe-bebê servirá de base para que o bebê construa um determinado tipo de interação social com a mãe que, por sua vez, servirá de modelo para o estabelecimento de futuras relações interpessoais da criança, ao longo de seu desenvolvimento nas diferentes situações sociais. Além disso, gradualmente, a criança constrói uma imagem coerente de si e do mundo através da reciprocidade que estabelece na relação com a mãe. Assim sendo, o comportamento da mãe serve como modelo para que a criança desenvolva um determinado comportamento interativo ao receber feedbacks de seus próprios comportamentos (Field, 1997; Murray \& Cooper, 1997).

A situação de risco do bebê prematuro ou a termo que necessita de cuidado intensivo neonatal, assim como o tempo de permanência do bebê na Unidade de Tratamento Intensivo Neonatal (UTINEO) pode despertar na mãe sentimentos de intensa culpa e profunda tristeza, prejudicando o surgimento do sentimento de competência materna e, conseqüentemente, de sensibilidade materna (Klaus \& Kennell, 1993). Na etiologia da prematuridade, são encontrados como determinantes principais, os fatores demográficos (raça, idade, educação e estado civil), os fatores médicos anteriores à gravidez (abortos, natimortos ou doenças), os fatores médicos associados à gravidez atual (sangramento vaginal, infecções ou pouco aumento de peso) e os fatores comportamentais e ambientais pré-natais (nutrição precária, cuidado inadequado no prénatal, fumo, uso de álcool e drogas, exposição a estresse ou a substâncias tóxicas). Contudo, muitos desses fatores estão inter-relacionados e a condição socioeconômica influi em quase todos (Papalia \& Olds, 2000).

Além dos problemas clínicos orgânicos enfrentados pelos recém-nascidos prematuros e a termo de risco, são observadas dificuldades no que se refere à interação entre os pais e seus bebês. Enquanto alguns bebês parecem hiperativos e hiperexcitáveis, outros podem parecer apáticos e sonolentos, quase não reagindo às tentativas de comunicação dos pais, até mesmo pelo fato da capacidade de interação e de regulação do estado de vigília de bebês nascidos pré-termo ser avaliada como inferiores pelo exame de Brazelton (escala de avaliação do comportamento neonatal) se comparados com recémnascidos a termo sadios que não necessitaram de tratamento intensivo neonatal (Mazet \& Stoleru, 1990). Assim sendo, a comunicação entre os pais e recém-nascidos prematuros e a termo de risco é observada como mais difícil de ser estabelecida, se comparada com a comunicação entre os pais e recém-nascidos sadios. As dificuldades na comunicação envolvendo as trocas interativas podem prejudicar o desenvolvimento da sensibilidade materna e, conseqüentemente, o estabelecimento da interação recíproca e sintônica entre a díade mãe-bebê (Mazet \& Stoleru, 1990).

O evento do nascimento pré-termo, por si só, pode ser vivenciado pelos pais como uma crise que desafia todas as metas e expectativas que eles tiveram durante o período de gestação (Maldonado, 1990; Soifer, 1980). As reações mais freqüentes entre as mães de bebês pré-termo são sentimentos de fracasso, inferioridade, culpa e tristeza, explicando o nascimento precoce de seu bebê com base em um defeito pessoal, o qual acreditam ser físico. Além disso, a separação precoce entre a mãe e o bebê, após o parto, pode aumentar ainda mais os sentimentos de menosvalia, reforçando a fantasia de sua inadequação como mãe. Em 
alguns casos, o bebê prematuro é percebido pela mãe como incompleto, ou de qualidade inferior pelo baixo peso e pela aparência física frágil (Klaus \& Kennell, 1993). É comum que essas mães se acusem por terem sido más, por terem exposto o filho à forte tensão, forçando-os para fora de seu útero protetor ou por algum mal feito, sendo o nascimento prétermo tido como uma punição ou castigo (Mazet \& Stoleru, 1990). Tais sentimentos podem ser compreendidos uma vez que o processo de separação psicológica, que os pais realizam durante os últimos três meses da gravidez para receber seu filho é interrompido pelo nascimento precoce. Para Klaus e Kennell (1993), a criança prematura possui pais também prematuros, o que pode dificultar ainda mais a formação do apego, tendo em vista o conceito de sensibilidade materna.

Estudos têm mostrado que o aleitamento materno pode aumentar a oportunidade de conhecimento e intimidade entre mãe e filho, e que a estimulação tátil durante o aleitamento é um componente essencial para a interação da díade mãe-bebê. O contato físico entre a mãe e o bebê durante a amamentação é fundamental para o aprendizado do bebê sobre a sua mãe, assim como para a construção de um relacionamento íntimo entre eles (Cunnigham, Jellife, \& Jellife, 1991). Segundo Klaus, Kennell e Klaus (2000), as mães que amamentam ao peito relatam que a cada amamentação têm uma renovada sensação de proximidade, calor e amor, aumentando a ligação com o seu bebê. Um estudo realizado na Inglaterra observou dois grupos de mães e bebês em que foram comparados os comportamentos maternos de mães que amamentavam ao peito e de mães que não amamentavam ao peito. Através deste estudo foi constatado que as mães que amamentavam seus filhos ao peito tenderam a tocar, acariciar e sorrir mais, ficando mais atentas ao processo de pausa-sucção do bebê em comparação às mães que alimentavam seus bebês com mamadeira (Lavelli \& Poli, 1998), mostrando-se mais sensíveis aos sinais do bebê.

A troca de olhares entre mãe-bebê também é uma das modalidades interativas essenciais, a qual se constitui pelo contato olho no olho, desde os primeiros dias de vida do bebê. Neste sentido, a amamentação pode ser considerada como uma das oportunidades de trocas afetivas, pois o olhar do bebê recém-nascido tem efeitos importantes sobre a mãe, representando um exemplo dos processos bidirecionais de interação. O olhar recíproco da mãe aumenta a tendência do bebê fixar os olhos sobre o rosto da mãe. Enquanto alguns bebês engajam-se muito ativamente nos episódios de olhares mútuos, outros estabelecem um contato visual menos prolongado e menos intenso. As mães desempenham um papel fundamental na qualidade e na duração desses episódios, respondendo de diferentes formas ao olhar de seu bebê (Mazet \& Stoleru, 1990). O olhar mútuo é uma forma poderosa de comunicação social, a qual pode ser experimentada durante a situação de amamentação (Stern, 1992). Aos dois ou três meses de vida, o bebê busca o olhar mútuo mais avidamente do que em outras etapas do desenvolvimento. O sorriso social está instalado e as vocalizações começam a ser dirigidas aos outros. Os bebês assumem o controle de início, manutenção, término e evitação do contato visual nas atividades sociais, que promovem o desenvolvimento do sentido de competência e de controle voluntário do bebê. Para Brazelton e Cramer (1992) estes dois elementos são essenciais para o desenvolvimento da autonomia do bebê. Stern (1992) acrescenta, afirmando que a autonomia do bebê opera de forma inerente em todos os comportamentos sociais da criança, regulando a quantidade e a qualidade do contato desde o nascimento. Para Stern, o período entre dois e seis meses é talvez o momento de vida mais exclusivamente social, sendo a amamentação uma das maneiras de se observar a interação, assim como a sensibilidade materna.

A alimentação do bebê recém-nascido também é vista como o primeiro momento de interação social, no qual o processo de pausa-sucção, caracterizado por pegar e largar, sincronicidade e reciprocidade entre mãe e bebê, representa a primeira ocorrência de diálogo da díade mãe-bebê. Nesse sentido, o presente estudo tem como objetivo verificar a relação entre o tipo de alimentação do bebê e os comportamentos interativos da mãe, especialmente a sensibilidade e o envolvimento materno em díades com recémnascidos prematuros e a termo de risco com necessidade de tratamento intensivo neonatal, e em díades com recémnascidos a termo sadios que não necessitaram de nenhum tipo de tratamento intensivo neonatal.

\section{Método}

\section{Participantes}

Este estudo envolveu a participação de 58 díades de mães e bebês com idade gestacional entre 32 e 42 semanas obtida conforme o método de New Ballard. Todas as díades foram avaliadas quando o bebê completava dois meses de idade cronológica ou corrigida, para os recém-nascidos prétermo ( $M=61,8$ dias, $D P=3,5$ dias), informação obtida por meio do prontuário do bebê. As díades mãe-bebê foram distribuídas em três grupos conforme a idade gestacional e a necessidade de tratamento intensivo neonatal: Grupo I: 20 díades de mães e bebês nascido pré-termo com idade gestacional entre 32 e 36 semanas que necessitaram de tratamento intensivo neonatal (grupo pré-termo de risco); Grupo II: 14 díades de mães e bebês nascidos a termo com idade gestacional entre 37 e 42 semanas que necessitaram de tratamento intensivo neonatal (grupo a termo de risco); Grupo III: 24 díades de mães e bebês nascidos a termo com idade gestacional entre 37 e 42 semanas sem nenhum tipo de tratamento intensivo neonatal (grupo controle).

Os critérios utilizados para a classificação da necessidade de tratamento intensivo neonatal envolveram um ou mais dos seguintes itens: uso de oxigênio em campânula, terapia continuada de ar (CPAP), apnéia, tratamento para asfixia (moderada e severa), sépsis neonatal, tratamento para convulsão, sendo tais informações obtidas por meio do prontuário do bebê. Não foram incluídos bebês nascidos com 
alteração cromossômica, malformação congênita, HIV positivo, sífilis congênita e malformação cerebral, assim como mães com idade inferior a 16 anos e com história de doença mental ou internação psiquiátrica.

A Tabela 1 mostra as características dos participantes. Conforme o esperado, a idade gestacional e o peso ao nascer são significativamente menores no Grupo I (pré-termo de risco) do que nos demais grupos investigados $(p=0,000)$. Podese dizer que $57 \%$ dos pais possui o $1^{0}$ Grau Incompleto, renda familiar média de três salários mínimos ao mês, 95\% das mães fizeram pré-natal, e apenas 16\% das mães fizeram uso de álcool ou tabaco durante a gestação. A paridade foi igualmente distribuída entre os grupos, assim como o sexo do bebê, 90\% dos partos foram vaginais, $92 \%$ das mães possuem uma situação conjugal estável com seus parceiros e foi boa a aceitação da gravidez, na maioria dos casos (80\%).

Com relação ao Apagar no $5^{\circ}$ minuto de vida, os Grupos I e II (pré-termo e a termo de risco) apresentaram menores índices do que o Grupo Controle $(p=0,001)$. A idade do recém-nascido no momento da coleta de dados não diferiu entre os grupos, bem como a idade da mãe e a idade do pai. Entre os bebês que necessitaram de tratamento intensivo neonatal, o Grupo I (pré-termo de risco) permaneceu significativamente mais tempo hospitalizado do que o Grupo II (a termo de risco) $(p=0,015)$.

O tipo de alimentação do bebê diferiu significativamente entre os grupos ( $p=0,017)$. O Grupo I (pré-termo de risco) foi mais alimentado por fórmula, enquanto que os Grupos II e III (a termo de risco e controle, respectivamente) foram mais alimentados por leite materno.
As demais variáveis sócio-demográficas investigadas, como escolaridade dos pais, renda familiar, pré-natal, uso de álcool ou tabaco na gestação, paridade, tipo de parto, aceitação da gravidez, sexo do bebê e situação conjugal, não diferiram entre os grupos estudados.

Não houve associação entre as características dos participantes e o tipo de alimentação do bebê.

\section{Instrumento}

Para investigar os comportamentos de interação mãebebê foi utilizado o Protocolo de Observação da Interação Mãe-Bebê 0-6 Meses. Esse protocolo foi elaborado por Schermann, Bohlin e Hagekull (1994), tendo sido traduzido e adaptado para a língua portuguesa por Schermann, com o objetivo de analisar os comportamentos de interação entre mães e bebês. $\mathrm{O}$ instrumento constitui-se de 20 itens, sendo onze referentes ao comportamento da mãe, oito referentes ao comportamento do bebê e um referente ao comportamento da díade. Cada um dos itens do protocolo possui cinco opções de pontuação entre um e cinco, em ordem crescente, do menos satisfatório ao mais satisfatório, com descrição detalhada do comportamento correspondente. Também foi realizada uma entrevista estruturada com a mãe, a fim de conhecer melhor os aspectos sócio-demográficos das famílias.

\section{Procedimentos}

Os dados foram coletados no Ambulatório de Seguimento Neonatal do Hospital São Lucas da PUCRS quando os bebês contavam com dois meses de idade cronológica e corrigida para a prematuridade. As díades do Grupo III (gru-

Tabela 1

Caracterização dos participantes

\begin{tabular}{|c|c|c|c|c|c|c|c|c|c|c|c|c|}
\hline & \multicolumn{3}{|c|}{$\begin{array}{l}\text { Grupo I } \\
(n=20)\end{array}$} & \multicolumn{3}{|c|}{$\begin{array}{l}\text { Grupo II } \\
(\mathrm{n}=14)\end{array}$} & \multicolumn{3}{|c|}{$\begin{array}{c}\text { Grupo III } \\
(\mathrm{n}=24)\end{array}$} & \multirow[b]{2}{*}{$F$} & \multirow[b]{2}{*}{$p$} & \multirow[b]{2}{*}{ Tukey HSD } \\
\hline & $M$ & $D P$ & (Min-Max) & $M$ & $D P$ & (Min-Max) & $M$ & $D P$ & (Min-Max) & & & \\
\hline Idade RN (dias) & 62,40 & 4,08 & $(53-68)$ & 61,57 & 3,96 & (54-68) & 61,71 & 2,65 & $(56-68)$ & 0,30 & 0,743 & \\
\hline Idade Gestacional (semanas) & 33,65 & 1,42 & $(32-36)$ & 39,43 & 1,34 & $(37-42)$ & 39,21 & 1,18 & $(37-41)$ & 122,13 & 0,000 & $1<2,3$ \\
\hline Apagar $5 \mathrm{~min}$. & 8,25 & 0,91 & $(6-9)$ & 8,29 & 1,54 & $(5-10)$ & 9,33 & 0,76 & $(7-10)$ & 7,38 & 0,001 & $1,2<3$ \\
\hline Peso ao nascer (gramas) & 2007 & 444,47 & $(1315-3070)$ & 3034 & 659,84 & $(1560-3920)$ & 3288 & 428,83 & $(2650-4260)$ & 38,44 & 0,000 & $1<2,3$ \\
\hline Idade mãe (anos) & 24,30 & 8,90 & $(16-41)$ & 26,36 & 6,54 & $(17-38)$ & 23,88 & 5,69 & $(16-37)$ & 0,57 & 0,572 & \\
\hline \multirow[t]{2}{*}{ Idade pai (anos) } & 28,53 & 10,25 & $(17-48)$ & 32,71 & 7,97 & $(23-47)$ & 26,00 & 6,32 & $(16-40)$ & 2,97 & 0,060 & \\
\hline & $M$ & $D P$ & (Min-Max) & $M$ & $D P$ & (Min-Max) & & & & $t$ & $p$ & \\
\hline Tempo do RN na UTINEO & 20,50 & 13,71 & $(2-53)$ & 11,07 & 7,61 & $(3-33)$ & & & & 2,563 & 0,015 & \\
\hline Tipo de alimentação do bebê & Freq. & $\%$ & & Freq. & $\%$ & & Freq. & $\%$ & & $\div 2$ & $p$ & \\
\hline Leite materno & 3 & 15 & & 8 & 57,1 & & 14 & 58,3 & & 11,717 & 0,017 & \\
\hline Fórmula & 11 & 55 & & 4 & 28,6 & & 4 & 16,7 & & & & \\
\hline Mista & 6 & 30 & & 2 & 14,3 & & 6 & 25 & & & & \\
\hline
\end{tabular}

Nota: Grupo I: pré-termo de risco; Grupo II: a termo de risco; Grupo III: controle

$\mathrm{M}=$ média, $\mathrm{DP}=$ desvio padrão, Min-Max = Mínimo e Máximo, $\mathrm{F}=$ análise de variância, $\mathrm{t}=$ teste $\mathrm{t}$ de Student. 
po controle) foram recrutadas do alojamento conjunto do referido hospital. Das 60 díades selecionadas, 30 compareceram ao Ambulatório, e seis foram excluídas, devido às idades da mãe e do bebê.

Na primeira etapa da coleta de dados, era lido aos pais o consentimento livre e esclarecido com todas as informações necessárias à participação no estudo. A partir do consentimento, era realizada a filmagem da interação mãe-bebê em situação face-a-face, em que se solicitava à mãe que brincasse com o bebê da maneira como ela estava acostumada a fazer em casa durante o período de três minutos consecutivos. Para tanto, o bebê era posicionado deitado na mesa de exame, direcionado à mãe que permanecia em pé e à frente da criança. A câmera de vídeo era colocada em um tripé estático, focalizando o bebê e a mãe lateralmente. Após o início da filmagem, o examinador permanecia atrás da díade (fora do foco de visão) como forma de minimizar a sua possível interferência. Todas as filmagens de interação mãebebê foram pontuadas por uma observadora treinada e sem nenhum conhecimento do grupo no qual os sujeitos se inseriam. Previamente, foi realizado o teste de acordo entre observadores em que a média global entre os comportamentos da mãe, da criança e da díade foi $83,17 \%$, o que denota um percentual de acordo satisfatório.

Os dados foram analisados de forma quantitativa através do programa Statistical Package for Social Sciences (SPSS, versão Windows 11,0). Foram utilizados testes estatísticos descritivos e inferenciais para diferença entre as médias e comparação entre grupos e testes correlacionais. O nível de significância adotado foi de $5 \%$.

\section{Resultados}

Os itens do protocolo de observação da interação, quando analisados isoladamente através do teste estatístico de Kruskall Wallis, mostraram diferenças significativas e tendências somente em relação aos comportamentos interativos da mãe, conforme mostra a Tabela 2. Observa-se que as mães do Grupo III (grupo controle) são mais atentas à criança $(p=0,005)$, tendem a estabelecer mais contato visual $(p=0,056)$ e tendem a expressar mais afeto positivo ( $p=$ $0,088)$ e menos afeto negativo $(p=0,089)$, respondendo mais aos comportamentos sociais do bebê $(p=0,061)$ durante a interação, do que as mães do Grupo I e II de bebês que necessitaram de tratamento intensivo neonatal. Os itens do bebê e a sintonia da interação não atingiram diferenças significativas entre os grupos (Tabela 2).

Para visualizar as diferenças referentes aos comportamentos da mãe durante a interação de forma mais consistente, os itens referentes ao comportamento materno foram categorizados em duas variáveis: sensibilidade materna e envolvimento materno conforme proposto por Schermann, Hagekull, Bohlin, Persson e Sedin (1997). Os itens referentes à variável sensibilidade incluíram a resposta da mãe ao comportamento social do bebê, a sensibilidade geral e a atenção geral da mãe, os quais indicam aspectos qualitativos do comportamento materno durante a interação. Os itens agrupados em envolvimento materno foram: freqüência de verbalização, quantidade de contato visual, expressão de afeto positivo, expressão de afeto negativo, que referem aspectos quantitativos do comportamento materno. O coeficiente Alfa (de Cronbach) para a sensibilidade materna foi 0,88 e para o envolvimento materno foi 0,84 . Estes valores indicam uma satisfatória consistência das categorias.

A sensibilidade materna diferiu entre os três grupos investigados ( $p=0,039$ ), havendo uma maior incidência de sensibilidade materna no Grupo III (grupo controle). Com relação ao envolvimento materno, foi constatada uma tendência à significância ( $p=0,071)$, que segue a mesma direção, em que as mães de bebês nascidos a termo e sadios são mais sensíveis aos sinais de seus bebês, tendendo a um maior envolvimento durante a interação do que mães de bebês nascidos pré-termo ou a termo de risco (Tabela 3).

Tabela 2

Itens do protocolo de interação mãe-bebê em relação aos grupos

\begin{tabular}{|c|c|c|c|c|c|c|c|c|c|c|c|}
\hline & & $\begin{array}{l}\text { Grup } \\
(\mathrm{n}=\end{array}$ & & & $\begin{array}{l}\text { Gru } \\
(\mathrm{n}=\end{array}$ & & & $\begin{array}{c}\text { Gru } \\
\text { (n }\end{array}$ & & & \\
\hline & $M$ & $D P$ & (Min-Max) & $M$ & $D P$ & (Min-Max) & $M$ & $D P$ & (Min-Max) & $K W$ & $p$ \\
\hline Contato visual & 3,80 & 0,77 & $3-5$ & 3,57 & 0,51 & 3-4 & 4,13 & 0,68 & $3-5$ & 5,775 & 0,056 \\
\hline Expressão afeto positivo & 2,60 & 1,10 & $1-5$ & 2,93 & 1,07 & $1-5$ & 3,25 & 0,79 & $2-4$ & 4,868 & 0,088 \\
\hline Resposta comportamento social & 3,45 & 0,94 & $2-5$ & 3,21 & 0,70 & $2-4$ & 3,83 & 0,64 & $3-5$ & 5,592 & 0,061 \\
\hline Expressão afeto negativo & 3,95 & 0,69 & $1-3$ & 4,00 & 0,68 & $1-3$ & 4,37 & 0,71 & $1-3$ & 4,827 & 0,089 \\
\hline Atenção geral & 3,35 & 0,93 & $2-5$ & 3,50 & 0,94 & $2-5$ & 4,21 & 0,78 & $2-5$ & 10,653 & 0,005 \\
\hline
\end{tabular}

Nota: Grupo I: pré-termo de risco; Grupo II: a termo de risco; Grupo III: controle

$\mathrm{M}=$ média, $\mathrm{DP}$ = desvio padrão, Min-Max = Mínimo e Máximo, $\mathrm{KW}=$ Kruskal Wallis. 
Tabela 3

Sensibilidade e envolvimento materno em relação aos grupos

\begin{tabular}{lcccccccccccc}
\hline & \multicolumn{3}{c}{$\begin{array}{l}\text { Grupo I } \\
\end{array}$} & \multicolumn{3}{c}{$(\mathrm{n}=20)$} & & \multicolumn{2}{c}{$\begin{array}{l}\text { Grupo II } \\
(\mathrm{n}=14)\end{array}$} & & \multicolumn{3}{c}{$\begin{array}{c}\text { Grupo III } \\
(\mathrm{n}=24)\end{array}$} \\
\hline & $M$ & $D P$ & (Min-Max) & $M$ & $D P$ & (Min-Max) & $M$ & $D P$ & (Min-Max) & $K W$ & $p$ \\
\hline Sensibilidade & 9,50 & 2,91 & $5-15$ & 9,86 & 2,25 & $6-14$ & 11,25 & 2,11 & $6-14$ & 6,479 & 0,039 \\
Envolvimento & 16,50 & 3,93 & $10-24$ & 17,29 & 3,20 & $10-23$ & 18,86 & 2,73 & $13-23$ & 5,286 & 0,071 \\
\hline
\end{tabular}

Nota: Grupo I: pré-termo de risco; Grupo II: a termo de risco; Grupo III: controle

$\mathrm{M}$ = média, $\mathrm{DP}$ = desvio padrão, Min-Max = Mínimo e Máximo, $\mathrm{KW}=$ Kruskal Wallis.

Dos comportamentos interativos da mãe, observados durante a interação mãe-bebê, a sensibilidade materna foi a única variável que diferiu quanto ao tipo de alimentação do bebê $(p=0,048)$. Mães que amamentam seus bebês exclusivamente com leite materno apresentam uma sensibilidade mais satisfatória do que as mães que não amamentam exclusivamente ao peito.

\section{Discussão e conclusões}

O presente estudo procurou verificar a relação entre o tipo de alimentação do bebê e os comportamentos de interação mãe-bebê em díades com recém-nascidos prematuros (Grupo I) e a termo de risco (Grupo II) com necessidade de tratamento intensivo neonatal, e em díades com recém-nascidos a termo sadios (Grupo III), que não necessitaram de nenhum tipo de tratamento intensivo neonatal.

Quando itens referentes ao comportamento materno, avaliados no Protocolo de Observação da Interação da Mãe-Bebê, foram analisados isoladamente, observou-se que as mães do Grupo III (controle) apresentaram maior atenção com relação a seus bebês durante a situação de interação do que as mães dos demais grupos investigados. Igualmente, foi constatada uma tendência das mães do Grupo III (controle) demonstrarem mais contato visual, expressarem mais afeto positivo e menos afeto negativo, bem como responderem mais aos comportamentos sociais da criança, do que as mães do Grupo I (pré-termo de risco) e Grupo II (a termo de risco). Da mesma forma, a sensibilidade materna foi mais satisfatória entre as mães do Grupo III (controle) do que nos demais grupos, apesar do Grupo II também ter sido alimentado por leite materno. Possivelmente, isso possa ser explicado pelo nascimento de risco, e não pelo tipo de alimentação. Estes resultados confirmam a teoria do apego no que se refere à importância do contato inicial entre a díade mãe-bebê desde o nascimento para a formação do apego. A proximidade entre mãe e filho favorece aos comportamentos interativos propiciados pela troca de olhares, expressão de afeto positivo, atenção geral, resposta aos comportamentos sociais do bebê e amamentação, assim como o despertar da sensibilidade ma- terna na medida em que a mãe de um bebê sadio pode experimentar continuamente tais comportamentos. Dessa forma, estas mães possuem maiores chances de exercitar suas capacidades em perceber, interpretar e responder adequadamente às necessidades da criança, sendo estas capacidades fundamentais para a formação do apego seguro entre mãe-bebê, de acordo com Bowlby (1990).

Com relação aos recém-nascidos pré-termo e a termo de risco (Grupos I e II) poderia se pensar que, de acordo com os achados deste estudo, estes se encontram, no momento da avaliação, mais prejudicados em termos do desenvolvimento social do que os recém-nascidos do Grupo III (controle), devido à menor incidência de aleitamento materno, bem como um maior tempo de permanência na UTINEO, mesmo com a sua idade cronológica dos recémnascidos de risco corrigida para a prematuridade. Dessa forma, o impacto do nascimento de risco e a necessidade de atendimento intensivo neonatal do bebê parecem ter repercussões na interação, pelo menos, até os bebês do presente estudo completarem dois meses de idade.

$\mathrm{O}$ fato de as mães que amamentam seus bebês apresentarem uma sensibilidade mais satisfatória durante a interação atenta para os benefícios do aleitamento materno no âmbito da relação da díade. Este resultado confirma os achados de estudos anteriores indicando que o contato entre mãe e bebê durante o aleitamento materno é um importante fator mediador das relações posteriores entre mãe e filho (Field, 1997; Klaus \& Kennell, 1993). A partir disso, é essencial que mesmo em recém-nascidos pré-termo e a termo de risco o aleitamento materno seja incentivado, pois pode minimizar os efeitos negativos do nascimento prematuro e de risco em termos relacionais e psicológicos.

De acordo com Klaus e Kennell (1993), o afastamento causado pela necessidade de cuidados intensivos neonatais, associado ao próprio evento do nascimento prematuro, prejudica na formação do apego materno e, conseqüentemente, a formação de novos vínculos afetivos estabelecidos pela criança em suas relações interpessoais futuras.

Os dados do presente estudo não apontam diferenças 
entre os grupos e os escores dos comportamentos interativos da criança e da díade. Este resultado não é de todo surpreendente, uma vez que outros estudos mostram evidências semelhantes, especialmente, nas fases iniciais do desenvolvimento da criança. É possível que estas diferenças apareçam posteriormente, quando os comportamentos sociais da criança tornam-se mais evidentes e observáveis. Schermann et al. (1997) realizaram um estudo longitudinal em que os comportamentos interativos da criança diferiram nos grupos estudados apenas quando as crianças atingiram a idade de quatro e seis meses.

A partir dos resultados do presente estudo pode-se pensar que o impacto do parto prematuro, bem como a necessidade da criança permanecer sob cuidados intensivos neonatais, esteja contribuindo para que estas mães mostremse menos sensíveis aos sinais de seus filhos, não sendo capazes de perceber, interpretar e responder adequadamente às necessidades da criança.

Estes resultados adquirem importância na medida em que a literatura traz dados bastante consistentes que apontam a sensibilidade materna como um elemento facilitador no desenvolvimento mental, motor e comportamental da criança ao longo de seu crescimento (Field, 1997).

A partir do estudo realizado, é possível inferir que a sensibilidade materna é mais favorável em mães de bebês nascidos a termo e sadios do que em mães de bebês nascidos com risco neonatal. O aleitamento materno é um importante fator capaz de facilitar o estabelecimento de uma sensibilidade materna mais favorável, sendo fundamental o incentivo da amamentação mesmo para recém-nascidos pré-termo e a termo de risco. Conforme o esperado no momento da avaliação, os recém-nascidos de risco, principalmente os bebês prematuros e não amamentados ao peito parecem estar em desvantagem em comparação com os bebês a termo que não necessitam de tratamento intensivo neonatal no que se refere aos comportamentos maternos que denotam sensibilidade.

\section{Referências}

Ainsworth, M. D. (1982). Attachment: retrospect and prospect. In C. M. Parkes \& J. S. Hinde (Orgs.), The place of attachment in human behavior (pp. 0330). Nova York: Basic Books.

Bowlby, J. (1990). Apego. São Paulo: Martins Fontes.

Brazelton, T., \& Cramer, B. (1992). As primeiras relações. São Paulo: Martins Fontes.

Cunnigham, A. S., Jellife, D. B., \& Jellife, E. F. (1991). Breastfeeding and health in the 1980s: a global epidemiologic review. The Journal of Pediatrics, 118, 659-666.

Field, T. (1997). The treatment of depressed mothers and their infants. In L. Murray \& P. Cooper (Orgs.), Postpartum depression and child development (pp. 221-236). Nova York: Guilford.

Klaus, M. H., \& Kennell, J. H. (1993). Pais/bebê: a formação do apego. Porto Alegre: Artes Médicas.

Klaus, M. H., Kennell, J. H., \& Klaus, P. H. (2000). Vínculo - construindo as bases para um apego seguro. Porto Alegre: Artes Médicas.

Lavelli, M., \& Poli, M. (1998). Early mother-infant interaction during breast and bottle-feeding. Infant Behavior and Development, 21, 667-684.

Maldonado, M. T. (1990). Psicologia da gravidez: parto e puerpério. Petrópolis: Vozes.

Mazet, P., \& Stoleru, S. (1990). Manual de psicopatologia do recém-nascido. Porto Alegre: Artes Médicas.

Murray, L., \& Cooper, P. (1997). The role of infant and maternal factors in postpartum depression, mother-infant interactions, and infant outcomes. In L. Murray \& P. Cooper (Orgs.), Postpartum depression and child development (pp. 111-135). Nova York: Guilford.

Papalia, D. E., \& Olds, S. W. (2000). Desenvolvimento físico nos três primeiros anos de vida. In D. E. Papalia \& S. W. Olds (Orgs.), Desenvolvimento humano (pp. 91-123). Porto Alegre: Artes Médicas.

Schermann, L., Hagekull, B., Bohlin, G., Persson, K., \& Sedin, G. (1997) Interaction between mother and infant born at risk during the first six months of corrected age. Acta Paediatrica, 86, 864-872.

Schermann, L., Bohlin, G., \& Hagekull, B. (1994). Interaction between mother and pre-term infant at 34 weeks post-conceptional age. Early Development and Parenting, 3, 171-180.

Soifer, R. (1980). Psicologia da gravidez: parto e puerpério. Porto Alegre: Artes Médicas.

Stern, D. (1992). O mundo interpessoal do bebê. Porto Alegre: Artes Médicas.

Cristiane Alfaya, mestre em Psicologia Clínica pela Pontifícia Universidade Católica (RS), é doutoranda em Psicologia pela Universidade Federal do Rio Grande do Sul e professora na Universidade Luterana do Brasil, campus de Santa Maria. Endereço para correspondência: Av. Marechal Andrea, 05, apto 107; Porto Alegre, RS; 91340-400. E-mail: c.alfaya@terra.com.br

Ligia Shermann, doutora em Psicologia Clínica pela Pontifícia Universidade Católica de São Paulo, é professora da Pós-graduação em Saúde Coletiva da Universidade Luterana do Brasil, campus de Canoas, RS. 